\title{
Improved Estimation of Rare Sensitive Attribute in a Stratified Sampling Using Poisson Distribution
}

\author{
Abdul Wakeel, Masood Anwar \\ Department of Mathematics, COMSATS Institute of Information Technology, Islamabad, Pakistan \\ Email: chabdulwakeel@gmail.com
}

Received 21 December 2015; accepted 20 February 2016; published 23 February 2016

Copyright $@ 2016$ by authors and Scientific Research Publishing Inc.

This work is licensed under the Creative Commons Attribution International License (CC BY).

http://creativecommons.org/licenses/by/4.0/

(c) (i) Open Access

\begin{abstract}
In this study, we propose a two stage randomized response model. Improved unbiased estimators of the mean number of persons possessing a rare sensitive attribute under two different situations are proposed. The proposed estimators are evaluated using a relative efficiency comparison. It is shown that our estimators are efficient as compared to existing estimators when the parameter of rare unrelated attribute is known and in unknown case, depending on the probability of selecting a question.
\end{abstract}

Keywords

Poisson Distribution, Rare Sensitive Attribute, Rare Unrelated Attribute, Stratified Sampling

\section{Introduction}

The collection of data through direct questioning on rare sensitive issues such as extramarital affairs, family disturbances and declaring religious affiliation in extremism condition is far-reaching issue. Warner [1] introduced the randomized response procedure to procure trustworthy data for estimating $\pi$, the proportion of respondents in the population belonging to the sensitive group. Greenberg et al. [2] suggested an unrelated question randomized response model in which each individual selected in the samples was asked to reply "yes" or " $n$ " to one of two statements: (a) Do you belong to Group A? (b) Do you belong to Group $Y$ ? with respective probabilities $P$ and $(1-P)$. Second question asked in the sampling does not have any effect on the first question. Greenberg et al. [2] considered $\pi_{A}$ and $\pi_{Y}$ the proportion of persons possessing sensitive and unrelated characteristic respectively and discussed both the cases when $\pi_{Y}$ was known and unknown. The probability of yes 
responses $\theta_{0}$, defined by them is $\theta_{0}=P \pi_{A}+(1-P) \pi_{Y}$. Mangat and Singh [3] proposed a two stage randomized response procedure which required the use of two randomization devices. The random device $R_{1}$ consists of two statements namely (a) I belong to the sensitive group, and (b) Go to random device $R_{2}$, with probabilities $T$ and $(1-T)$ respectively. The random device $R_{2}$ which uses two statements (a) I belong to the sensitive group, and (b) I do not belong to the sensitive group with known probabilities $P$ and $(1-P)$ respectively. Then $\theta_{0}$, the probability of yes responses is $\theta_{0}=T \pi+(1-T)\{P \pi+(1-P)(1-\pi)\}$.

Later on, different modifications have been made to improve the methodology for collection of information. Some of them are Lee et al. [4], Chaudhuri and Mukerjee [5], Mahmood et al. [6], Land et al. [7], Bhargava and Singh [8].

Land et al. [7] proposed the estimators for the mean number of persons possessing the rare sensitive attribute using the unrelated question randomized response model by utilizing a Poisson distribution. Recently, Lee et al. [4] extended the Land et al.'s [7] study to stratify sampling and propose the estimators when the parameter of rare unrelated attribute is known and unknown.

In this study, we propose improved estimators for the mean and its variance of the number of persons possessing a rare sensitive attribute based on stratified sampling by using Poisson distribution. The estimators are proposed when the parameter of the rare unrelated attribute is known and unknown. The proposed estimators are evaluated using a relative efficiency comparing the variances of the estimators reported in Lee et al. [4].

\section{Improved Estimation of a Rare Sensitive Attribute in Stratified Sampling-Known Rare Unrelated Attributes}

Consider the population of size $N$ individuals which is divided into $L$ subpopulations (strata) of sizes $N_{h}(h=1,2, \cdots, L)$. All the subpopulations are disjoint and together comprise the whole population. In stratum $h$, $n_{h}$ respondent are selected by simple random sampling with replacement (SRSWR) and asked to use the pair of randomization devices $R_{h 1}$ and $R_{h 2}$, each consisting of the two statements. The randomization device $R_{h 1}$ is constructed as:

(i) "I possessrare sensitive attribute $A$ "

(ii) "Go to randomization device $R_{h 2}$ "

with respective probabilities $P_{1 h}$ and $\left(1-P_{1 h}\right)$.

The randomization device $R_{h 2}$ consists of two statements:

(i) "I possess rare sensitive attribute $A$ "

(ii) "I possess rare unrelated attribute $Y$ "

with probabilities $P_{2 h}$ and $\left(1-P_{2 h}\right)$ respectively.

By this randomized device, the probability of a yes response in stratum $h$ is given by

$$
\theta_{h 0}=P_{h 1} \pi_{h A}+\left(1-P_{h 1}\right)\left\{P_{h 2} \pi_{h A}+\left(1-P_{h 2}\right) \pi_{h Y}\right\},
$$

where $\pi_{h A}$ and $\pi_{h Y}$ are the population proportions of individuals possessing rare sensitive and rare unrelated attributes in the $h^{\text {th }}$ stratum, respectively. Here $\pi_{h Y}$ is assumed to be known. Since $A$ and $Y$ are very rare attributes, $n_{h} \theta_{h 0}=\lambda_{h 0}$ is finite, assuming $n_{h} \rightarrow \infty$ and $\theta_{h 0} \rightarrow 0$.

Let $x_{h 1}, x_{h 2}, \cdots, x_{h h_{h}}$ be an $n_{h}$ random sample in stratum $h$ from a Poisson distribution with parameter $\lambda_{h 0}$. Then the maximum likelihood estimator for the mean number of persons who have the rare sensitive attribute in stratum $h, \lambda_{h A}\left(=n_{h} \pi_{h A}\right)$, is given by

$$
\hat{\lambda}_{h A}=\frac{1}{\left\{P_{h 1}+\left(1-P_{h 1}\right) P_{h 2}\right\}}\left[\frac{1}{n_{h}} \sum_{i=1}^{n_{h}} x_{h i}-\left(1-P_{h 1}\right)\left(1-P_{h 2}\right) \lambda_{h Y}\right],
$$

where $\lambda_{h Y}=n_{h} \pi_{h Y}$ is (known) mean of persons who have rare unrelated attribute in stratum $h$. The parameter $\lambda_{A}$, is the mean number of persons possessing rare sensitive attribute $A$, in a population of size $N$ and its estimator $\hat{\lambda}_{A}$ is given by

$$
\hat{\lambda}_{A}=\sum_{h=1}^{L} W_{h} \hat{\lambda}_{h A}=\sum_{h=1}^{L} W_{h} \frac{1}{\left\{P_{h 1}+\left(1-P_{h 1}\right) P_{h 2}\right\}}\left[\frac{1}{n_{h}} \sum_{i=1}^{n_{h}} x_{h i}-\left(1-P_{h 1}\right)\left(1-P_{h 2}\right) \lambda_{h Y}\right],
$$

where $W_{h}=N_{h} / N$. 
The variance of the estimator $\hat{\lambda}_{h A}$ in each stratum is given by

$$
V\left(\hat{\lambda}_{h A}\right)=\frac{A_{h}}{n_{h}}
$$

where

$$
A_{h}=\frac{\lambda_{h A}}{\left[P_{h 1}+\left(1-P_{h 1}\right) P_{h 2}\right]}+\frac{\left(1-P_{h 1}\right)\left(1-P_{h 2}\right) \lambda_{h Y}}{\left[P_{h 1}+\left(1-P_{h 1}\right) P_{h 2}\right]^{2}} .
$$

Thus, the variance expression of the estimator $\hat{\lambda}_{A}$ may be derived as

$$
V\left(\hat{\lambda}_{A}\right)=V\left[\sum_{h=1}^{L} W_{h} \hat{\lambda}_{h A}\right]=\sum_{h=1}^{L} \frac{W_{h}^{2} A_{h}}{n_{h}} .
$$

THEOREM 1. $\hat{\lambda}_{A}$ is an unbiased estimator of $\lambda_{A}$.

Proof. From (3), we have

$$
\begin{aligned}
E\left(\hat{\lambda}_{A}\right) & =\sum_{h=1}^{L} \frac{W_{h}}{\left[P_{h 1}+\left(1-P_{h 1}\right) P_{h 2}\right]}\left\{\frac{1}{n_{h}} \sum_{i=1}^{n_{h}} E\left(x_{h i}\right)-\left(1-P_{h 1}\right)\left(1-P_{h 2}\right) \lambda_{h Y}\right\}, \\
& =\sum_{h=1}^{L} W_{h}\left\{\frac{\lambda_{h 0}-\left(1-P_{h 1}\right)\left(1-P_{h 2}\right) \lambda_{h Y}}{P_{h 1}+\left(1-P_{h 1}\right) P_{h 2}}\right\}=\sum_{h=1}^{L} W_{h} \lambda_{h A}=\lambda_{A} .
\end{aligned}
$$

THEOREM 2. The unbiased estimator for $V\left(\hat{\lambda}_{A}\right)$ is given by

$$
\hat{V}\left(\hat{\lambda}_{A}\right)=\sum_{h=1}^{L} \frac{W_{h}^{2}}{n_{h}^{2}\left[P_{h 1}+\left(1-P_{h 1}\right) P_{h 2}\right]^{2}}\left\{\sum_{i=1}^{n_{h}} x_{h i}\right\} .
$$

Proof.

$$
\begin{aligned}
E\left\{\hat{V}\left(\hat{\lambda}_{A}\right)\right\} & =\sum_{h=1}^{L} \frac{W_{h}^{2}}{n_{h}^{2}\left[P_{h 1}+\left(1-P_{h 1}\right) P_{h 2}\right]^{2}}\left\{\sum_{i=1}^{n_{h}} E\left(x_{h i}\right)\right\} \\
& =\sum_{h=1}^{L} \frac{W_{h}^{2}}{n_{h}\left[P_{h 1}+\left(1-P_{h 1}\right) P_{h 2}\right]^{2}}\left\{\lambda_{h 0}\right\}=V\left(\hat{\lambda}_{A}\right)
\end{aligned}
$$

Now, we consider the proportional and optimal allocations of the total sample size $n$ into different strata. The method of proportional allocation is used to define sample sizes in each stratum depending on each stratum size. Since the sample size in each stratum is defined as $n_{h}=n N_{h} / N$, the variance of the estimator $\hat{\lambda}_{A}$, under proportional allocation of sample size is given by

$$
V\left(\hat{\lambda}_{A}\right)_{\text {prop }}=\frac{1}{n}\left[\sum_{h=1}^{L} W_{h} A_{h}\right] .
$$

However, the optimal allocation is a technique to define sample size to minimize variance for a given cost or to minimize the cost for a specified variance. The $n_{h}$ is proportionate to the standard deviation, $S_{h}$ of the variable. In stratified sampling, let cost function is defined as $C=c_{0}+\sum_{h=1}^{L} c_{h} n_{h}$, where $c_{0}$ is the fixed cost and $c_{h}$ is the cost for the each individual stratum. Within each stratum the cost is proportional to the size of sample, but the cost $c_{h}$ may vary from stratum to stratum. For fixed cost, using the Cauchy Schwarz inequality, the sample size $n_{h}$ to minimize $V\left(\hat{\lambda}_{A}\right)$ is given by

$$
n_{h}=n \times \frac{W_{h} \sqrt{A_{h} / c_{h}}}{\sum_{h=1}^{L} W_{h} \sqrt{A_{h} / c_{h}}} .
$$

So the minimum variance of the estimator for the specified cost $C$ under the optimum allocation of sample 
size is given by

$$
V\left(\hat{\lambda}_{A}\right)_{o p t}=\frac{1}{n}\left[\sum_{h=1}^{L} W_{h} \sqrt{A_{h} \times c_{h}}\right] \times\left[\sum_{h=1}^{L} W_{h} \sqrt{A_{h} / c_{h}}\right] .
$$

\section{Improved Estimation of a Rare Sensitive Attribute in Stratified Sampling-Unknown Rare Unrelated Attributes}

In this section, the estimators for the mean number of rare sensitive attribute are proposed under the assumptions that the sizes of stratum are known; however, $\lambda_{h Y}=n_{h} \pi_{h Y}$, the mean of the rare unrelated attribute is unknown. In this case each selected respondent from stratum $h$ is asked to use the sequential pair of randomization devices. That in the $h^{\text {th }}$ stratum, $n_{h}$, respondents are asked to use the randomization devices $R_{h 1}$ and $R_{h 2}$ consisting of two statements. The device $R_{h 1}$ consists of two statements:

(i) "I possess a sensitive group $A$ "

(ii) "Go to randomization device $R_{h 2}$ "

The statements occur with respective probabilities $P_{1 h}$ and $\left(1-P_{1 h}\right)$.

The two statements of the randomization device $R_{h 2}$ are:

(i) "I possess a sensitive attribute $A$ "

(ii) "I possess unrelated attribute $Y$ "

represented with respective probabilities $P_{2 h}$ and $\left(1-P_{2 h}\right)$. After using the first pair of randomized devices, respondent is asked to use the same pair of devices $R_{h 1}$ and $R_{h 2}$ but with probabilities $T_{1 h},\left(1-T_{1 h}\right)$ and $T_{2 h},\left(1-T_{2 h}\right)$, respectively.

The probabilities of the yes responses for the first and second use of pair of randomization devices are respectively given by

$$
\theta_{h 1}=P_{h 1} \pi_{h A}+\left(1-P_{h 1}\right)\left[P_{h 1} \pi_{h A}+\left(1-P_{h 1}\right) \pi_{h Y}\right]
$$

and

$$
\theta_{h 2}=T_{h 1} \pi_{h A}+\left(1-T_{h 1}\right)\left[T_{h 1} \pi_{h A}+\left(1-T_{h 1}\right) \pi_{h Y}\right],
$$

where $\pi_{h A}$ and $\pi_{h Y}$ are the respective population proportions of rare sensitive and rare unrelated attribute in the stratum $h$. As $n_{h}$ is large and $\left(\pi_{h A}, \pi_{h Y}\right) \rightarrow 0$, therefore $\left(\theta_{h 1}, \theta_{h 2}\right) \rightarrow 0$. Now, obviously $\lambda_{h 1}=n_{h} \theta_{h 1}, \lambda_{h 2}$ $=n_{h} \theta_{h 2}$. Let $x_{h 1 i}$ and $x_{h 2 i}\left(h=1,2, \cdots, L, \quad i=1,2, \cdots, n_{h}\right)$ be the pair of responses from the $i$ th respondent selected in $h^{\text {th }}$ stratum. We have

$$
\begin{gathered}
\operatorname{Var}\left(x_{h 1 i}\right)=E\left(x_{h 1 i}\right)=\lambda_{h 1}=P_{h 1} \lambda_{h A}+\left(1-P_{h 1}\right)\left\{P_{h 2} \lambda_{h A}+\left(1-P_{h 2}\right) \lambda_{h Y}\right\} \\
\operatorname{Var}\left(x_{h 2 i}\right)=E\left(x_{h 2 i}\right)=\lambda_{h 2}=T_{h 1} \lambda_{h A}+\left(1-T_{h 1}\right)\left\{T_{h 2} \lambda_{h A}+\left(1-T_{h 2}\right) \lambda_{h Y}\right\} \\
\operatorname{Cov}\left(x_{h 1}, x_{h 2 i}\right)=E\left(x_{h 1 i} x_{h 2 i}\right)-E\left(x_{h 1 i}\right) E\left(x_{h 2 i}\right) \\
=\left\{P_{h 1}+\left(1-P_{h 1}\right) P_{h 2}\right\}\left\{T_{h 1}+\left(1-T_{h 1}\right) T_{h 2}\right\} \lambda_{h A}+\left(1-P_{h 1}\right)\left(1-P_{h 2}\right)\left(1-T_{h 1}\right)\left(1-T_{h 2}\right) \lambda_{h Y},
\end{gathered}
$$

Following the expression given in Equations (12) and (13), we have the sample means for both set of responses as

$$
\frac{1}{n_{h}} \sum_{i=1}^{n_{h}} x_{h 1 i}=P_{h 1} \hat{\lambda}_{h A}+\left(1-P_{h 1}\right)\left[P_{h 2} \hat{\lambda}_{h A}+\left(1-P_{h 2}\right) \hat{\lambda}_{h Y}\right]
$$

and

$$
\frac{1}{n_{h}} \sum_{i=1}^{n_{h}} x_{h 2 i}=T_{h 1} \hat{\lambda}_{h A}+\left(1-T_{h 1}\right)\left[T_{h 2} \hat{\lambda}_{h A}+\left(1-T_{h 2}\right) \hat{\lambda}_{h Y}\right] .
$$

By solving (15) and (16), we get estimators of $\lambda_{h A}$ and $\lambda_{h Y}$ as

$$
\hat{\lambda}_{h A}=\frac{1}{n_{h} B_{h}}\left[\left(1-T_{1 h}\right)\left(1-T_{2 h}\right) \sum_{i=1}^{n_{h}} x_{h 1 i}-\left(1-P_{1 h}\right)\left(1-P_{2 h}\right) \sum_{i=1}^{n_{h}} x_{h 2 i}\right]
$$




$$
\hat{\lambda}_{h Y}=\frac{1}{n_{h} D_{h}}\left[\left\{T_{1 h}+\left(1-T_{1 h}\right) T_{2 h}\right\} \sum_{i=1}^{n_{h}} x_{h 1 i}-\left\{P_{1 h}+\left(1-P_{1 h}\right) P_{2 h}\right\} \sum_{i=1}^{n_{h}} x_{h 2 i}\right]
$$

where

$$
\begin{aligned}
B_{h}=\left\{P_{h 1}+\left(1-P_{h 1}\right) P_{h 2}\right\} & -\left\{T_{h 1}+\left(1-T_{h 1}\right) T_{h 2}\right\} \text { and } D_{h}=\left\{T_{h 1}+\left(1-T_{h 1}\right) T_{h 2}\right\}-\left\{P_{h 1}+\left(1-P_{h 1}\right) P_{h 2}\right\} . \\
V\left(\hat{\lambda}_{h A}\right)= & \frac{1}{\left[n_{h} B_{h}\right]^{2}} V\left[\left(1-T_{h 1}\right)\left(1-T_{h 2}\right) \sum_{i=1}^{n_{h}} x_{h 1 i}-\left(1-P_{h 1}\right)\left(1-P_{h 2}\right) \sum_{i=1}^{n_{h}} x_{h 2 i}\right], \\
= & \frac{1}{\left[n_{h} B_{h}\right]^{2}}\left[\left(1-T_{1 h}\right)^{2}\left(1-T_{2 h}\right)^{2} \sum_{i=1}^{n_{h}} V\left(x_{h 1 i}\right)+\left(1-P_{1 h}\right)^{2}\left(1-P_{2 h}\right)^{2} \sum_{i=1}^{n_{h}} V\left(x_{h 2 i}\right)\right. \\
& \left.-2\left(1-T_{1 h}\right)\left(1-T_{2 h}\right)\left(1-P_{1 h}\right)\left(1-P_{2 h}\right) \sum_{i=1}^{n_{h}} \operatorname{Cov}\left(x_{h 1 i}, x_{h 2 i}\right)\right]
\end{aligned}
$$

Puttinng (12), (13) and (14) in (19) we get

$$
V\left(\hat{\lambda}_{h A}\right)=\sum_{h=1}^{L} \frac{\left[A_{h 1}+A_{h 2}\right]}{n_{h} B_{h}^{2}},
$$

where

$$
\begin{aligned}
A_{h 1}= & {\left[\left(1-T_{h 1}\right)^{2}\left(1-T_{h 2}\right)^{2}\left\{P_{h 1}+\left(1-P_{h 1}\right) P_{h 2}\right\}+\left(1-P_{h 1}\right)^{2}\left(1-P_{h 2}\right)^{2}\left\{T_{h 1}+\left(1-T_{h 1}\right) T_{2}\right\}\right.} \\
& \left.-2\left(1-T_{h 1}\right)\left(1-T_{h 2}\right)\left(1-P_{h 1}\right)\left(1-P_{h 2}\right)\left\{T_{h 1}+\left(1-T_{h 1}\right) T_{h 2}\right\}\left\{P_{h 1}+\left(1-P_{h 1}\right) P_{h 2}\right\}\right] \lambda_{h A}, \\
A_{h 2}= & {\left[\left(1-T_{h 1}\right)\left(1-T_{h 2}\right)\left(1-P_{h 1}\right)\left(1-P_{h 2}\right)\left\{2-\left(T_{h 1}+\left(1-T_{h 1}\right) T_{h 2}\right)-\left(P_{h 1}+\left(1-P_{h 1}\right) P_{h 2}\right)\right\}\right.} \\
& \left.-2\left\{\left(1-T_{h 1}\right)\left(1-T_{h 2}\right)\left(1-P_{h 1}\right)\left(1-P_{h 2}\right)\right\}^{2}\right] \lambda_{h Y} .
\end{aligned}
$$

The stratified estimators of $\lambda_{A}$ and $\lambda_{Y}$ are defined as

$$
\hat{\lambda}_{A}=\sum_{h=1}^{L} W_{h} \hat{\lambda}_{h A} \text {, and } \hat{\lambda}_{Y}=\sum_{h=1}^{L} W_{h} \hat{\lambda}_{h Y}
$$

THEOREM 3. $\hat{\lambda}_{A}$ is an unbiased estimator for $\lambda_{A}$.

Proof.

$$
\begin{aligned}
E\left(\hat{\lambda}_{A}\right) & =E\left[\sum_{h=1}^{L} W_{h} \hat{\lambda}_{h A}\right]=\sum_{h=1}^{L} \frac{W_{h}}{n_{h} B_{h}}\left[\left(1-T_{1 h}\right)\left(1-T_{2 h}\right) \sum_{i=1}^{n_{h}} E\left(x_{h 1 i}\right)-\left(1-P_{1 h}\right)\left(1-P_{2 h}\right) \sum_{i=1}^{n} E\left(x_{h 2 i}\right)\right] \\
& =\sum_{h=1}^{L} \frac{W_{h}}{B_{h}}\left[\left(1-T_{1 h}\right)\left(1-T_{2 h}\right) \lambda_{h 1}-\left(1-P_{1 h}\right)\left(1-P_{2 h}\right) \lambda_{h 2}\right] .
\end{aligned}
$$

Putting the values of $\lambda_{h 1}$ and $\lambda_{h 2}$ in Equation (22), we get the result.

THEOREM 4. The variance of $\hat{\lambda}_{A}$ is given by

where

$$
V\left(\hat{\lambda}_{A}\right)=\sum_{h=1}^{L} \frac{W_{h}^{2}}{n_{h} B_{h}^{2}}\left[A_{h 1}+A_{h 2}\right]
$$

$$
\begin{aligned}
A_{h 1}= & {\left[\left(1-T_{h 1}\right)^{2}\left(1-T_{h 2}\right)^{2}\left\{P_{h 1}+\left(1-P_{h 1}\right) P_{h 2}\right\}+\left(1-P_{h 1}\right)^{2}\left(1-P_{h 2}\right)^{2}\left\{T_{h 1}+\left(1-T_{h 1}\right) T_{2}\right\}\right.} \\
& \left.-2\left(1-P_{h 1}\right)\left(1-P_{h 2}\right)\left(1-T_{h 1}\right)\left(1-T_{h 2}\right)\left\{P_{h 1}+\left(1-P_{h 1}\right) P_{h 2}\right\}\left\{T_{h 1}+\left(1-T_{h 1}\right) T_{h 2}\right\}\right] \lambda_{h A}, \\
A_{h 2}= & {\left[\left(1-P_{h 1}\right)\left(1-P_{h 2}\right)\left(1-T_{h 1}\right)\left(1-T_{h 2}\right)\left\{2-\left(P_{h 1}+\left(1-P_{h 1}\right) P_{h 2}\right)-\left(T_{h 1}+\left(1-T_{h 1}\right) T_{h 2}\right)\right\}\right.} \\
& \left.-2\left\{\left(1-P_{h 1}\right)\left(1-P_{h 2}\right)\left(1-T_{h 1}\right)\left(1-T_{h 2}\right)\right\}^{2}\right] \lambda_{h Y} .
\end{aligned}
$$


Proof. Since $\hat{\lambda}_{A}=\sum_{h=1}^{L} W_{h} \hat{\lambda}_{h A}$, we have

$$
V\left(\hat{\lambda}_{A}\right)=V\left[\sum_{h=1}^{L} W_{h} \hat{\lambda}_{h A}\right]=\sum_{h=1}^{L} W_{h}^{2} V\left(\hat{\lambda}_{h A}\right)
$$

On putting (20) in (24) we have the theorem.

Corollary 1: An unbiased estimator for the variance of rare sensitive attribute is given by

$$
\hat{V}\left(\hat{\lambda}_{A}\right)=\sum_{h=1}^{L} \frac{W_{h}^{2}}{n_{h} B_{h}^{2}}\left[\hat{A}_{h 1}+\hat{A}_{h 2}\right]
$$

It can be proved easily.

THEOREM 5. $\hat{\lambda}_{Y}$ is an unbiased estimator of $\lambda_{Y}$.

Proof. From (18), we have

$$
\begin{aligned}
& E\left(\hat{\lambda}_{h Y}\right) \\
= & \frac{1}{n_{h}\left[\left\{T_{h 1}+\left(1-T_{h 1}\right) T_{h 2}\right\}-\left\{P_{h 1}+\left(1-P_{h 1}\right) P_{h 2}\right\}\right]}\left[\left\{T_{h 1}+\left(1-T_{h 1}\right) T_{h 2}\right\} \sum_{i=1}^{n_{h}} E\left(x_{h 1 i}\right)-\left\{P_{h 1}+\left(1-P_{h 1}\right) P_{h 2}\right\} \sum_{i=1}^{n_{h}}\left(x_{h 2 i}\right)\right] \\
= & \frac{1}{\left[\left\{T_{h 1}+\left(1-T_{h 1}\right) T_{h 2}\right\}-\left\{P_{h 1}+\left(1-P_{h 1}\right) P_{h 2}\right\}\right]}\left[\left\{T_{h 1}+\left(1-T_{h 1}\right) T_{h 2}\right\} \lambda_{h 1}-\left\{P_{h 1}+\left(1-P_{h 1}\right) P_{h 2}\right\} \lambda_{h 2}\right] \\
= & \sum_{h=1}^{L} W_{h} E\left(\hat{\lambda}_{h Y}\right)=\sum_{h=1}^{L} W_{h} \lambda_{h Y}=\lambda_{Y} .
\end{aligned}
$$

Corollary 2: An unbiased estimator for $V\left(\hat{\lambda}_{Y}\right)$ is given by

$$
\hat{V}\left(\hat{\lambda}_{Y}\right)=\sum_{h=1}^{L} \frac{W_{h}^{2}}{n_{h} D_{h}^{2}}\left[C_{h 1} \hat{\lambda}_{h A}+C_{h 2} \hat{\lambda}_{h Y}\right]
$$

where

$$
\begin{aligned}
& C_{h 1}=[\left\{T_{h 1}+\left(1-T_{h 1}\right) T_{h 2}\right\}^{2}\left\{P_{h 1}+\left(1-P_{h 1}\right) P_{h 2}\right\}+\left\{P_{h 1}+\left(1-P_{h 1}\right) P_{h 2}\right\}^{2}\left\{T_{h 1}+\left(1-T_{h 1}\right) T_{h 2}\right\} \\
&\left.-2\left\{P_{h 1}+\left(1-P_{h 1}\right) P_{h 2}\right\}^{2}\left\{T_{h 1}+\left(1-T_{h 1}\right) T_{h 2}\right\}^{2}\right], \\
& C_{h 2}= {\left[\left\{T_{h 1}+\left(1-T_{h 1}\right) T_{h 2}\right\}^{2}\left(1-P_{h 1}\right)\left(1-P_{h 2}\right)+\left\{P_{h 1}+\left(1-P_{h 1}\right) P_{h 2}\right\}^{2}\left(1-T_{h 1}\right)\left(1-T_{h 2}\right)\right.} \\
&\left.-2\left\{P_{h 1}+\left(1-P_{h 1}\right) P_{h 2}\right\}\left\{T_{h 1}+\left(1-T_{h 1}\right) T_{h 2}\right\}\left(1-P_{h 1}\right)\left(1-P_{h 2}\right)\left(1-T_{h 1}\right)\left(1-T_{h 2}\right)\right], \\
& D_{h}=\left\{T_{h 1}+\left(1-T_{h 1}\right) T_{h 2}\right\}-\left\{P_{h 1}+\left(1-P_{h 1}\right) P_{h 2}\right\} .
\end{aligned}
$$

Now under proportional allocation of sample size, the variance of $\hat{\lambda}_{A}$ is given by

$$
V\left(\hat{\lambda}_{A}\right)_{\text {prop }}=\frac{1}{n} \sum_{h=1}^{L} \frac{W_{h}}{B_{h}^{2}}\left[A_{h 1}+A_{h 2}\right] .
$$

However, in optimum allocation, the sample size in stratum $h$ is

$$
n_{h}=n \times\left[\frac{W_{h}}{B_{h}} \sqrt{\left(A_{h 1}+A_{h 2}\right) / c_{h}}\right] \div\left[\sum_{h=1}^{L} \frac{W_{h}}{B_{h}} \sqrt{\left(A_{h 1}+A_{h 2}\right) / c_{h}}\right]
$$

and the variance of $\hat{\lambda}_{A}$ is given by

$$
V\left(\hat{\lambda}_{A}\right)_{\text {opt }}=\frac{1}{n}\left[\sum_{h=1}^{L} \frac{W_{h}}{B_{h}} \sqrt{\left(A_{h 1}+A_{h 2}\right) c_{h}}\right] \times\left[\sum_{h=1}^{L} \frac{W_{h}}{B_{h}} \sqrt{\left(A_{h 1}+A_{h 2}\right) / c_{h}}\right] .
$$




\section{Relative Efficiency}

Lee et al. [4] proposed variance of $\hat{\lambda}_{A}$ for rare sensitive attribute based on Poisson distribution when the rare unrelated attribute known and unknown respectively is:

$$
\begin{gathered}
V_{L 1}\left(\hat{\lambda}_{A}\right)=\sum_{h=1}^{L} \frac{W_{h}^{2}}{n_{h}}\left[\frac{\lambda_{h A}}{P_{h 1}}+\frac{\left(1-P_{h 1}\right) \lambda_{h y}}{P_{h 1}^{2}}\right], \\
V_{L 2}\left(\hat{\lambda}_{A}\right)=\sum_{h=1}^{L} \frac{W_{h}^{2}}{n_{h}\left(P_{h 1}-T_{h 1}\right)^{2}}\left[\Lambda_{h 1}+\Lambda_{h 2}\right],
\end{gathered}
$$

where

$$
\begin{aligned}
& \Lambda_{h 1}=\left\{P_{h 1}\left(1-T_{h 1}\right)^{2}+T_{h 1}\left(1-P_{h 1}\right)^{2}-2 P_{h 1} T_{h 1}\left(1-P_{h 1}\right)\left(1-T_{h 1}\right)\right\} \lambda_{h A} \\
& \Lambda_{h 2}=\left\{\left(1-P_{h 1}\right)\left(1-T_{h 1}\right)\left(2-P_{h 1}-T_{h 1}\right)-2\left(1-P_{h 1}\right)^{2}\left(1-T_{h 1}\right)^{2}\right\} \lambda_{h Y} .
\end{aligned}
$$

For comparison of the proposed estimator with $V_{L}\left(\hat{\lambda}_{A}\right)$, the relative efficiency is given by

$$
R E=\frac{V_{L}\left(\hat{\lambda}_{A}\right)}{V\left(\hat{\lambda}_{A}\right)} .
$$

Large samples are required to estimate the means of rare sensitive attribute. So we consider a large hypothetical population, in order to study the relative efficiency, setting $n=10000$ with two strata having $n_{1}=4000$ and $n_{2}=6000$. We choose values of the parameters $\left(\lambda_{1 A}, \lambda_{1 Y}\right),\left(\lambda_{2 A}, \lambda_{2 Y}\right)$ as $(0.5,1.5),(1.5,0.5),(1.5,1.5)$ and $(0.5,0.5)$, and we let the value $P_{12}$ range from 0.3 to 0.7 , and let that of $P_{11}$ range from 0.6 to 0.9 when the weights $W_{1}=0.4$ (and $W_{2}=0.6$ ) and $W_{1}=0.6$ (and $W_{2}=0.4$ ) which is proportional allocation. Also, let $\left(\lambda_{1 A}=\lambda_{2 A}\right)$ and $\left(\lambda_{1 Y}=\lambda_{2 Y}\right)$.

\subsection{Relative Efficiency When Rare Unrelated Attribute Is Known}

Let $V_{1}\left(\hat{\lambda}_{A}\right)$ be the variance of the proposed estimator $\hat{\lambda}_{A}$ for the rare sensitive attribute when the parameter of rare unrelated attribute is known. The relative efficiency of proposed estimator with respect to $V\left(\hat{\lambda}_{A}\right)_{L 1}$ esti-
mator is defined as

$$
R E_{1}=\frac{V\left(\hat{\lambda}_{A}\right)_{L 1}}{V_{1}\left(\hat{\lambda}_{A}\right)}=\frac{\left[\sum_{h=1}^{2} W_{h}\left\{\frac{\lambda_{h A}}{P_{h}}+\frac{\left(1-P_{h}\right) \lambda_{h Y}}{P_{h}^{2}}\right\}\right]}{\left[\sum_{h=1}^{2} W_{h}\left\{\frac{\lambda_{h A}}{\left[P_{h 1}+\left(1-P_{h 1}\right) P_{h 2}\right]}+\frac{\left(1-P_{h 1}\right)\left(1-P_{h 2}\right) \lambda_{h Y}}{\left[P_{h 1}+\left(1-P_{h 1}\right) P_{h 2}\right]^{2}}\right\}\right]} .
$$

From Equation (29) it evident that the relative efficiency of proposed estimator is free from the sample size $n$. We set the design probabilities as $P_{11}=P_{21}$ and $P_{12}=P_{22}$. In Table 1, the relative efficiencies are given with parameter values $\left(\lambda_{1 A}, \lambda_{1 Y}\right),\left(\lambda_{2 A}, \lambda_{2 Y}\right)$ as $(0.5,1.5),(1.5,0.5),(1.5,1.5)$ and $(0.5,0.5), P_{12}$ varies from 0.3 to 0.7 , and $P_{11}$ from 0.6 to 0.9 having weights $W_{1}=0.4,0.6 \quad\left(W_{1}+W_{2}=1\right)$. It is evident that the proposed estimator has efficiency greater than 1 in all cases, and is always better than the $V\left(\hat{\lambda}_{A}\right)_{L 1}$ estimator. A study of Figure 1 confirms this.

\subsection{Relative Efficiency When Rare Unrelated Attribute Is Unknown}

Let $V_{2}\left(\hat{\lambda}_{A}\right)$ be the variance of the proposed estimator $\hat{\lambda}_{A}$ for the rare sensitive attribute when the parameter of rare unrelated attribute is unknown. The relative efficiency of proposed estimator with respect to $V\left(\hat{\lambda}_{A}\right)_{L 2}$
estimator is defined as 

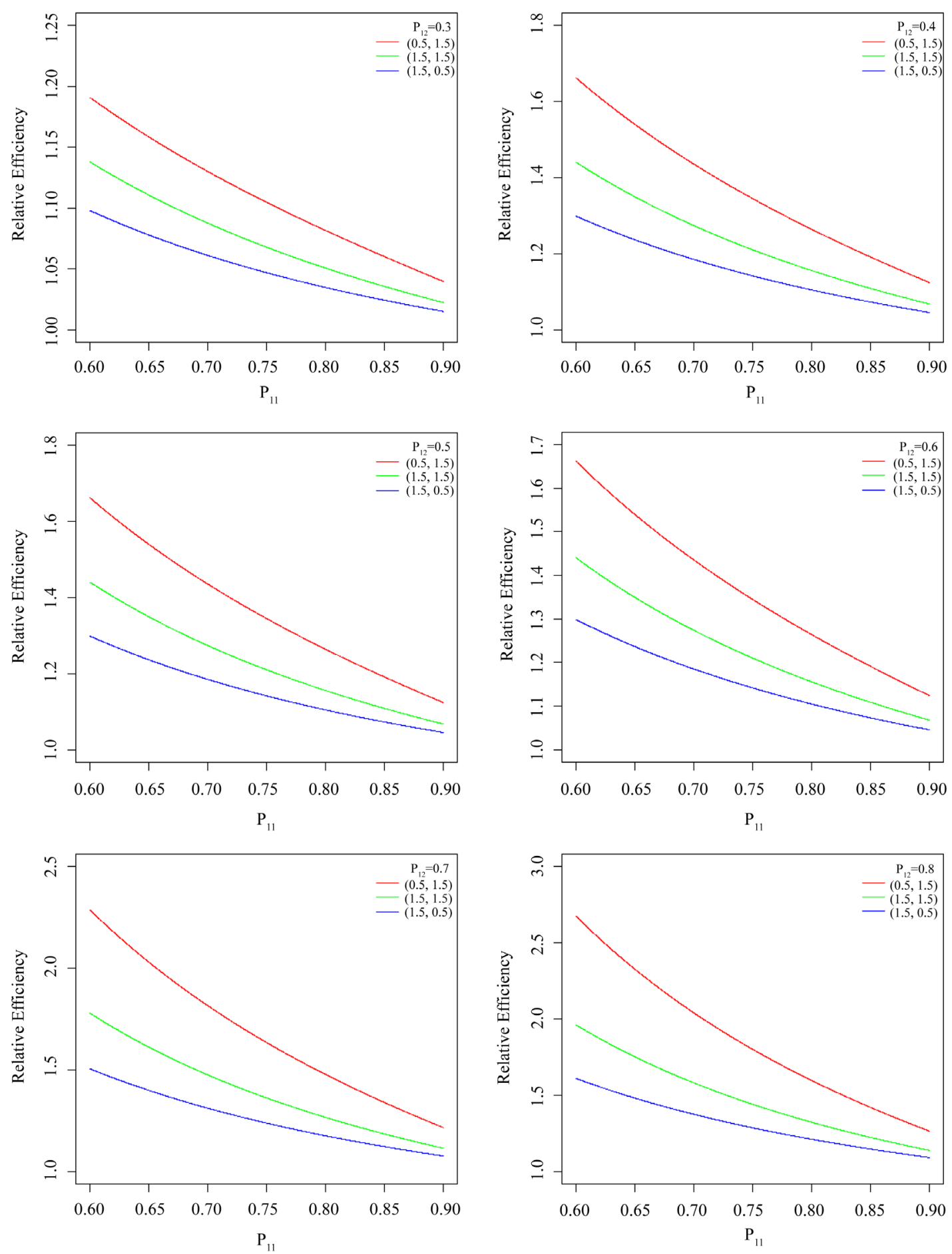

Figure 1. Relative Efficiency (RE) of the proposed model with respect to Lee et al. [4] for $W_{1}=0.4$ and $P_{12}=0.3$ to 0.8 .

$$
R E_{2}=\frac{V\left(\hat{\lambda}_{A}\right)_{L 2}}{V_{2}\left(\hat{\lambda}_{A}\right)}=\frac{\sum_{h=1}^{2} W_{h}\left[\frac{\left[A_{h 1}^{\prime}+A_{h 2}^{\prime}\right]}{\left[P_{h 1}-T_{h 1}\right]^{2}}\right.}{\sum_{h=1}^{2} W_{h} \frac{\left[A_{h 1}+A_{h 2}\right]}{\left[\left\{P_{h 1}+\left(1-P_{h 1}\right) P_{h 2}\right\}-\left\{T_{h 1}+\left(1-T_{h 1}\right) T_{h 2}\right\}\right]^{2}}} .
$$


The relative efficiency of proposed estimator is free from the sample size $n$. For the analysis, the design probabilities are fixed as $P_{11}=P_{21}, P_{12}=P_{22}, T_{11}=T_{21}, T_{12}=T_{22}$. Setting $\lambda_{1 A}=\lambda_{2 A}, \quad \lambda_{1 Y}=\lambda_{2 Y}$ with parameter values of $\left(\lambda_{1 A}, \lambda_{1 Y}\right),\left(\lambda_{2 A}, \lambda_{2 Y}\right)$ as $(0.5,1.5),(1.5,0.5),(1.5,1.5)$ and $P_{11}=0.6, T_{11}=0.3,0.4, T_{12}=0.2,0.3$, $0.4,0.5$ and $W_{1}=0.4,0.5 \quad\left(W_{1}+W_{2}=1\right)$. The relative efficiencies are given in Table 2 depict that the proposed estimator outer perform than $V\left(\hat{\lambda}_{A}\right)_{L 2}$ estimator having efficiency greater than 1 if we set the probabilities as $P_{12} \geq T_{12}$. However the relative efficiency starts decreasing as we take $P_{12}<T_{12}$. A study of Figure 2 confirms this. Also, when $W_{1}$ increasesthe relative efficiency of proposed estimator increases.

Table 1. Relative efficiency of the proposed estimator with Lee et al. (2013).

\begin{tabular}{|c|c|c|c|c|c|c|c|c|c|c|}
\hline \multirow[b]{2}{*}{$P_{12}$} & \multirow[b]{2}{*}{$\lambda_{1 Y}$} & \multirow[b]{2}{*}{$\lambda_{1 A}$} & \multicolumn{4}{|c|}{$W_{1}=0.4$} & \multicolumn{4}{|c|}{$W_{1}=0.6$} \\
\hline & & & $P_{11}=0.6$ & 0.7 & 0.8 & 0.9 & $P_{11}=0.6$ & 0.7 & 0.8 & 0.9 \\
\hline \multirow[t]{3}{*}{0.3} & 0.5 & 1.5 & 1.7346 & 1.5829 & 1.4758 & 1.3966 & 1.5630 & 1.4264 & 1.3299 & 1.2585 \\
\hline & 1.5 & 1.5 & 1.9238 & 1.7016 & 1.5439 & 1.4266 & 1.7336 & 1.5334 & 1.3912 & 1.2855 \\
\hline & 1.5 & 0.5 & 2.2198 & 1.9173 & 1.6887 & 1.5016 & 2.0003 & 1.7277 & 1.5217 & 1.3531 \\
\hline \multirow[t]{3}{*}{0.4} & 0.5 & 1.5 & 1.8713 & 1.6667 & 1.5228 & 1.4169 & 1.6863 & 1.5018 & 1.3723 & 1.2768 \\
\hline & 1.5 & 1.5 & 2.1435 & 1.8333 & 1.6166 & 1.4574 & 1.9316 & 1.6520 & 1.4567 & 1.3133 \\
\hline & 1.5 & 0.5 & 2.6070 & 2.1568 & 1.8251 & 1.5615 & 2.3492 & 1.9436 & 1.6447 & 1.4071 \\
\hline \multirow[t]{3}{*}{0.5} & 0.5 & 1.5 & 2.0097 & 1.7510 & 1.5701 & 1.4372 & 1.8109 & 1.5779 & 1.4148 & 1.2951 \\
\hline & 1.5 & 1.5 & 2.3751 & 1.9699 & 1.6908 & 1.4885 & $\begin{array}{c}2.2100 \\
402\end{array}$ & 1.7751 & 1.5327 & 1.3413 \\
\hline & 1.5 & 0.5 & 3.0537 & 2.4245 & 1.9727 & 1.6238 & 2.7517 & 2.1848 & 1.7776 & 1.4633 \\
\hline \multirow[t]{3}{*}{0.6} & 0.5 & 1.5 & 1.6090 & 1.01489 & 1.2107 & 1.0910 & 1.9370 & 1.6545 & 1.4576 & 1.3135 \\
\hline & 1.5 & 1.5 & 1.9600 & 1.4204 & 1.3225 & 1.1377 & 2.3596 & 1.9026 & 1.5921 & 1.3698 \\
\hline & 1.5 & 0.5 & 2.6727 & 1.6326 & 1.5961 & 1.2642 & 3.2177 & 2.4550 & 1.9215 & 1.5219 \\
\hline \multirow[t]{3}{*}{0.7} & 0.5 & 1.5 & 1.7147 & 1.4383 & 1.2464 & 1.1063 & 2.0642 & 1.7315 & 1.5005 & 1.3318 \\
\hline & 1.5 & 1.5 & 2.1511 & 1.6900 & 1.3806 & 1.1616 & 2.5897 & 2.0346 & 1.6621 & 1.3984 \\
\hline & 1.5 & 0.5 & 3.1223 & 2.2915 & 1.7258 & 1.3150 & 3.7592 & 2.7587 & 2.0776 & 1.5831 \\
\hline
\end{tabular}

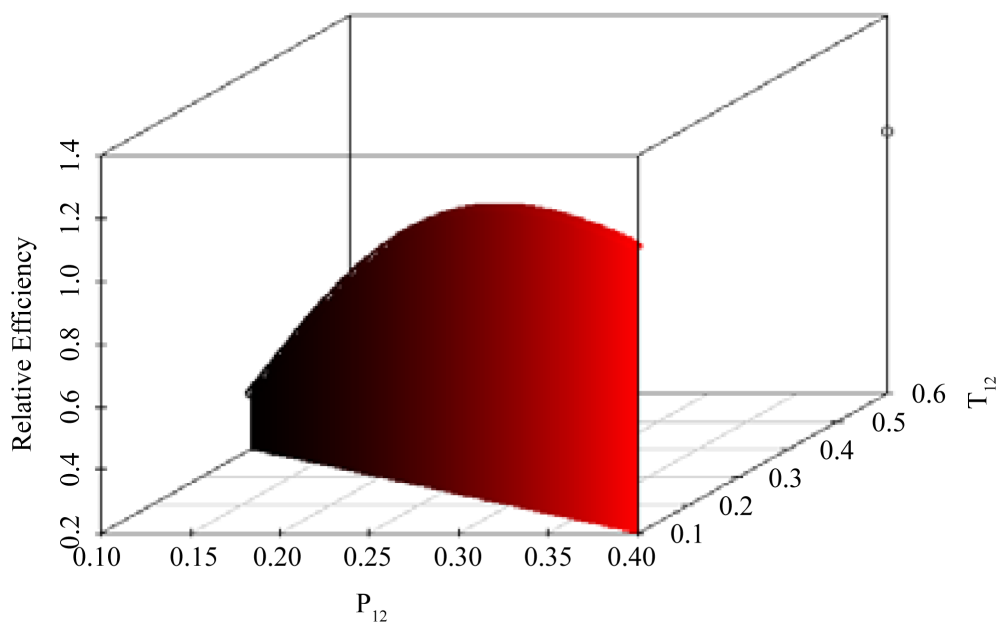

Figure 2. Relative Efficiency (RE) of the proposed model with respect to Lee et al. [4] for indicated values. 
Table 2. Relative efficiency of the proposed estimator with Lee et al. (2013), $W_{1}=0.4$, and $W_{1}=0.5$.

\begin{tabular}{|c|c|c|c|c|c|c|c|}
\hline$P_{11}=P_{21}$ & $P_{12}=P_{22}$ & $T_{11}=T_{21}$ & $T_{12}=T_{22}$ & $\lambda_{1 A}=\lambda_{2 A}$ & $\lambda_{1 Y}=\lambda_{2 Y}$ & $\operatorname{RE}\left(W_{1}=0.4\right)$ & $\operatorname{RE}\left(W_{1}=0.5\right)$ \\
\hline \multirow[t]{12}{*}{0.6} & 0.6 & 0.3 & 0.2 & 1.5 & 0.5 & 12.5971 & 15.7464 \\
\hline & & & & 1.5 & 1.5 & 16.9517 & 21.1896 \\
\hline & & & & 0.5 & 1.5 & 10.0051 & 12.5064 \\
\hline & & & 0.3 & 1.5 & 0.5 & 10.3926 & 12.9908 \\
\hline & & & & 1.5 & 1.5 & 13.9851 & 17.4814 \\
\hline & & & & 0.5 & 1.5 & 8.2542 & 10.3178 \\
\hline & & & 0.4 & 1.5 & 0.5 & 8.1881 & 10.2352 \\
\hline & & & & 1.5 & 1.5 & 11.0186 & 13.7732 \\
\hline & & & & 0.5 & 1.5 & 6.5033 & 8.1292 \\
\hline & & & 0.5 & 1.5 & 0.5 & 5.9836 & 7.4795 \\
\hline & & & & 1.5 & 1.5 & 8.0520 & 10.0651 \\
\hline & & & & 0.5 & 1.5 & 4.7524 & 5.9405 \\
\hline \multirow[t]{12}{*}{0.6} & 0.6 & 0.4 & 0.2 & 1.5 & 0.5 & 3.1703 & 3.9629 \\
\hline & & & & 1.5 & 1.5 & 4.4483 & 5.5603 \\
\hline & & & & 0.5 & 1.5 & 2.7607 & 2.4509 \\
\hline & & & 0.3 & 1.5 & 0.5 & 2.5759 & 3.2198 \\
\hline & & & & 1.5 & 1.5 & 3.6142 & 4.5178 \\
\hline & & & & 0.5 & 1.5 & 2.2431 & 2.8038 \\
\hline & & & 0.4 & 1.5 & 0.5 & 1.9814 & 2.4768 \\
\hline & & & & 1.5 & 1.5 & 2.7801 & 3.4752 \\
\hline & & & & 0.5 & 1.5 & 1.7254 & 2.1568 \\
\hline & & & 0.5 & 1.5 & 0.5 & 1.3870 & 1.7338 \\
\hline & & & & 1.5 & 1.5 & 1.9461 & 2.4326 \\
\hline & & & & 0.5 & 1.5 & 1.2078 & 1.5098 \\
\hline
\end{tabular}

\section{Conclusion}

In this study, a two stage randomized response model is proposed with improved estimators for the mean and its variance of the number of persons possessing a rare sensitive attribute based on stratified sampling by using Poisson distribution. It is shown that our proposed method have better efficiencies than the existing randomized response model, when the parameter of rare unrelated attribute is known and in unknown case, depending on the probability of selecting a question. For future work, we can obtain more sensitive information from respondents by using stratified double sampling with the proposed model.

\section{References}

[1] Warner, S.L. (1965) Randomized Response: A Survey Technique for Eliminating Evasive Answer Bias. Journal of 
Computational and Graphical Statistics, 60, 63-66. http://dx.doi.org/10.1080/01621459.1965.10480775

[2] Greenberg, B.G., Abul-Ela, A.L.A., Simmons, W.R. and Horvitz, D.G. (1969) The Unrelated Question Randomized Response Model: Theoretical Framework. Journal of the American Statistical Association, 64, 520-539. http://dx.doi.org/10.1080/01621459.1969.10500991

[3] Mangat, N.S. and Singh, R. (1990) On the Confidentiality Guaranteed under Randomized Response Sampling: A Comparison with Several New Techniques. Biometrical Journal, 40, 237-242.

[4] Lee, G.S., Uhm, D. and Kim, J.M. (2013) Estimation of a Rare Sensitive Attribute in a Stratified Sample Using Poisson Distribution. Statistics, 47, 685-709. http://dx.doi.org/10.1080/02331888.2011.625503

[5] Chaudhuri, A. and Mukerjee, R. (1988) Randomized Response: Theory and Techniques. Marcel Dekker, New York.

[6] Mahmood, M., Singh, S. and Horn, S. (1998) On the Confidentiality Guaranteed under Randomized Response Sampling: A Comparison with Several New Techniques. Biometrical Journal, 40, 237-242. http://dx.doi.org/10.1002/(SICI)1521-4036(199806)40:2<237::AID-BIMJ237>3.0.CO;2-N

[7] Land, M., Singh, S. and Sedory, S.A. (2012) Estimation of a Rare Sensitive Attribute Using Poisson Distribution. Statistics, 46, 351-360. http://dx.doi.org/10.1080/02331888.2010.524300

[8] Bhargava, M. and Singh, R. (2000) A Modified Randomization Device for Warner’s Model. Statistica, 60, $315-321$. 